\title{
DETERMINANTS OF THE PERCEIVED VERTICAL AND HORIZONTAL
}

\author{
BY JAMES J. GIBSON, Smith College
}

AND

O. H. MOWRER, Yale Untoersity

\section{INTRODUCTION}

The fact that the seen world appears upright is so obvious that it may seem axiomatic. Nevertheless this verticalhorizontal stability of phenomenal things is as much a psychological problem as their coloration, size, or distance. Stratton once asked the question, "why do things look right side up instead of inverted?" But a different and more fundamental question is, "why do things look right side up in the first place-instead of, for instance, tilted?" Some attention has been paid to the related observation that the objective visual field remains phenomenally upright when the head is tilted $(\mathbf{2 3}, \mathbf{3 0})$. But this phenomenon presupposes a more fundamental one, the fact that the objective visual field is phenomerially upright when the head is not tilted.

Koffka has recently described phenomenal space not only as a 'ground' for the perception of objects but also as a 'framework' with reference to which they are oriented and localized (23). Space, as experienced, has the quality of 'updownness' and 'right-leftness'; vertical and horizontal reference-axes are an implicit characteristic of all seeing. Or, in other words, we seem to possess a visual 'sense' of the vertical and horizontal. In support of this assertion, innumerable experiments have proved that we can imagine these axes in a perfectly homogeneous circular visual field, and can set an adjustable line in this field to the vertical or horizontal with great precision. This perceptual ability coexists with the ability to maintain and sense deviations from an erect posture, and also to feel the normality or tilt of the terrain and other 
environmental objects. Presumably these two sets of facts are the perceptual and the behavioral aspects of a common function. But the perceptual fact of a visual 'sense' of the vertical and the behavioral fact of postural orientation are not related in any simple way as cause and effect. There is evidence to show that, to a considerable degree, they are mutually dependant.

Gelb, for example, describes a patient with a brain injury who suffered from " a pathological change in the main directions of space" (I5). The visual environment, including houses, trees, and streets, appeared uniformly tilted to the right $\left(8^{\circ}-12^{\circ}\right)$ and a visual line looked upright (even with totally dark surroundings) only if it were objectively tilted to the left. The significant fact, however, was that the environment felt as well as looked tilted to the right; the patient's posture was objectively inclined to the left and only in this position did he feel himself erect. Gelb concludes that "the fact of pathological change in both the visual and the kinaesthetic-motor realms ... argues an underlying change of which these are symptoms." It is reasonable a priori that the visual and the kinæsthetic vertical should have to coincide, for the body after all is usually a visual object having a definite position and orientation in the visual field.

The sensory determinants of visual and postural uprightness have been rather thoroughly explored since the time of Mach. A large number of sensory cues have been shown to operate, divisible into two classes, first those arising from the pull of gravity upon the body and from the maintenance of postural equilibrium ( $g$-factors), and second those arising from purely visual stimulation ( $v$-factors). The first class includes excitations from the semicircular canals, utricle and saccule, and from the compensatory reactions to which they give rise; from bilateral variations in tactual pressure on the soles of the feet, buttocks, elbows, etc.; from the musclesense and the resulting tonic reflexes; and probably also from internal visceral pressures. The second class includes the retinal stimulation produced by the dominant lines of objects and surroundings in the visual world, that is to say the 
gravitational verticals and horizontals which nature and more especially civilization present to our eyes; and also the stimuli correlated with the familiar appearance of shapes not having such dominant lines (I9); and finally the shape of the boundary of the visual field formed by the nose, cheeks, and the supra-orbital ridges (7). The outlines of the nose, for example, are a part of every visual field and provide standard visual lines which might serve to fix axes of reference. These $v$-factors, it must be remarked, rest on the assumption that a fixed posture is maintained. The point will be expanded later.

It is obvious that these various stimulus-factors must interact in a complex way when one considers how they would be modified, sometimes concomitantly and sometimes not, in different situations. A considerable body of evidence has accumulated, both experimental and observational, bearing on the relative influence of the g-factors and of the $v$-factors in determining the visual vertical and also the postural vertical. Some of this evidence is conflicting. Koffka has proposed a bold theory to the effect that the phenomenal 'spatial framework' is constituted by the main lines of organization in the visual field; in other words the principal directions seen in this field create the frame of reference for it (23, chap. 6). This means of course that if a visual scene is artificially tilted, or even if its main lines (a window frame or the horizon) are inclined, the framework will follow and a new vertical will be established. The theory is consistently phenomenological, for it makes even the position of the body-the 'ego'-dependant on this visual framework. The ego, being only one of the objects in visual space, is assumed to be oriented in the same way as any other object-by reference to the main lines of visual organization.

There are indications to support such a theory of complete visual dominance but on the other hand there are many facts which contradict it. It is our purpose to evaluate some of this conflicting evidence and to propose a hypothesis: that 
both the visual and the postural vertical are determined by visual factors and gravitational factors acting jointly, with orientation to gravity, however, as the more decisive factor in cases of real confict between the two types of sensory data, and the primary factor genetically. We shall first examine the evidence for vision, and then the evidence for gravity.

The experiments and observations which we have to examine are of two sorts, first those in which the v-factors are modified or eliminated, as when the visual field is tilted or the subject is blindfolded, respectively, and second those in which the g-factors are modified, as when the pull of gravity is shifted by a centrifugal force. ${ }^{1}$ It is important to remember that we are concerned here with the elimination or shift of one kind of determinant relative to the other. There is a class of experiments related to this method and easy to confuse with it which is irrelevant to our particular problem. They have to do with the effect, or more generally the absence of effect, on the visual and kinæsthetic vertical of abnormal bodily postures, e.g., of tilting the head or body. In this situation one kind of determinant is not shifted relative to the other and they do not conflict; instead both are shifted concomitantly and by the same amount. From an objective point of view neither the pull of gravity nor the visual scene is shifted but only the subject's body and therefore the way in which these two affect his sense-organs. The seen and felt environment remain phenomenally upright when the head is tilted despite the change in the proximal stimuli; we are dealing, therefore, with a compensatory perceptual process of some sort-a type of constancy-phenomenon. Many experiments have verified the observation that in a dark room with only a single vertical line of light visible this constancy is only partial and the phenomenal direction of the line is a compromise between the objective and the retinal direction, but this fact, the Aubert phenomenon, must

1 It is clearly impossible to eliminate the gravitational determinants entirely, although Garten (x4) has in the absence of vision experimentally isolated several of them from one another. 
be regarded as a special case of a much more general tendency. ${ }^{2}$

In this view of the matter, the problem of the constancy of the perceptual framework, seen and felt, is to be distinguished from the prior problem of its sensory determinants and in particular the role played by vision. Koffka's 'visual organization' theory of the framework is eminently capable of explaining constancy, and was designed for this purpose. It remains to be seen whether it will stand up against experimental evidence of a simpler type.

\section{Evidence for the Dependance of the Vertical on Visual Determinants}

Elimination of visual cues.-That postural orientation is somewhat weakened in the dark, or when a subject is blindfolded, has been frequently noted On top of high buildings, on mountain sides, or in any unfamiliar homogeneous visual field where the usual lines of 'anchorage' are absent, normal posture is often reported as difficult to maintain, although it is possible that emotional in addition to purely sensory factors are at work here. Airplane pilots flying in clouds are reported to have emerged upside down without having been aware of their altered position. Failing vision is believed to be a frequent cause of the loss of balance in old age (2r). This evidence has even been formulated in the statement that "we stand with our eyes." The inference from these observations is that if the postural vertical is so dependant on visual cues, the visual vertical must be even more so. Strictly interpreted, however, these facts demonstrate no more than the occurrence of orientational errors under particular circumstances in the absence of $\mathrm{v}$-factors. They show that the latter are contributing determinants for orientation but not

${ }^{2}$ Investigations in this special field are complicated by the counter-rolling of the eyes in the head, which is however insufficient to account for either complete or partial constancy of the vertical, and by the fact that tilted posture may mean tilt of the head, of the trunk, or both. Müller has described the evidence in detail (30). The Aubert phenomenon is reported as highly variable and not reliable. One of the writers (Gibson) has never been able to observe it in a clear-cut manner; the luminous line in the dark usually appears 'really' vertical whatever the position of the head. 
that it can occur only when these are functioning. Moreover, as we shall see later, such observations are matched by experiments with precisely opposite implications-experiments in which the kinæsthetic and visual vertical prove to be very stable and quite precise in the absence of the $v$-factors.

Shift of the visual cues so as to conflict with gravity.Better proof of the contribution of $v$-factors is to be found in observations where the visual scene is tilted out of alignment with the axis of gravity. Hemholtz reported that passengers on shipboard see the cabin as stationary while a suspended barometer (i.e., a pendulum) seems to sway (38, p. 250). Innumerable landlubbers have observed the slow wheeling of the horizon beyond the fixed deck of the ship-and gone below in haste. The framework is undoubtedly anchored to the visual lines in this situation, but as Helmholtz himself went on to observe, the situation is only temporary. When the passenger gets his 'sea legs,' the ship seems to move and the barometer (or the horizon) remains fixed. The postural and the visual vertical for the veteran sailor are anchored to gravity.

A case where the ascendency of vision over gravity may seem even more striking is to be found in the 'haunted' or 'magic' swing, an amusement-park device. The 'swing' itself is usually wide enough to seat several persons side by side and is rigidly suspended from a heavy metal shaft passing horizontally through the center of a large, often elaborately furnished room. When the 'passengers' are seated and have been strapped in, they are told that they will be swung violently back and forth and that the swing will occasionally make a complete revolution. Actually the swing remains at rest, except perhaps for a little jostling; it is the room, with the furniture secured to the floor, that is put into motion. However, the illusion is quite compelling and persons often struggle violently to make the equilibritory adjustments which the visual stimulation seems to call for. The strapping of the 'passengers' to the seat is necessitated, not by the motion of the seat itself, but because of the danger that, in attempting to orient on the basis of the visual cues presented 
by the moving room, they may throw themselves out of the seat. In an article written in 1895 , Wood (40) makes the following significant comments regarding this illusion:

The curious and interesting feature however, was, that even though the action was fully understood, as it was in my case, it was impossible to quench the sensations of 'goneness within' with each apparent rush of the swing. The minute the eyes were shut the sensations vanished instantly. Many persons were actually made sick by the illusion. I have met a number of gentlemen who said they could scarcely walk out of the building from dizziness and nausea. I myself experienced no sensations of dizziness, being accustomed to heights and to rapid motion; but the sensation before described was always present (and I visited the place several times), though I tried to suppress it and reason against it.

These examples of a shift in the $\mathrm{v}$-factors are complicated by the fact that they involve motion. It is very doubtful that the 'magic swing' illusion would persist if the moving room were brought to rest in an oblique position. The occupants would surely recover an equilibrium based on gravity. Nevertheless the question arises, does the spatial framework attach itself to visual lines when these are objectively tilted but by an unchanging amount? Koff $k a$ believes that it does $(23$, p. 217). If one travels up a steep slope on a mountain railway and looks through the window of the railway car, the trees, telegraph poles and houses framed by the window often appear to be tilted rather than upright. The suggestion is that the car and the window frame dictate the framework and hence objective verticals and horizontals must necessarily look tilted.

Another illusory impression is that of the slope of a river flowing between canyon-walls composed of tilted rock-strata. A remarkable example is found in Ausable Chasm, New York, where the sightseer is taken downstream in a small boat. Here, the strata constituting the main lines of the visual field slope against the stream, and consequently the sightseer feels that he is 'shooting a rapids' at a fearsome degree of inclination. 
It is easy to assume in these examples that the principal visual lines which are objectively tilted (window, strata) straighten up completely and thereby inevitably cause the lesser lines in the field which are objectively upright (trees, houses, stream) to appear tilted by precisely the same invariant amount. But this assumption has apparently never been given any experimental verification. Possibly all that these observations make certain is that under given conditions upright visual objects look tilted, and this is not the same thing as saying that they do so because tilted objects look upright. There is in fact experimental evidence to contradict the assumption. If a subject looks through a wide paper cone at a visual field filled with inclined lines, there will be induced a simultaneous contrast effect on a single objectively vertical line in this field; that is to say, the single line will look tilted in the opposite direction. The significant fact is, however, that the inclined lines continue to look inclined even after prolonged inspection ( $\mathbf{7}$, p. 557). If the tilted visual field does not right itself under experimental conditions, it may not do so under uncontrolled conditions, given sufficiently attentive observation, and in this event our examples had better be ascribed to a contrast phenomenon rather than to a shift of the framework into alignment with the field. ${ }^{3}$

Wertheimer's experiment.-The strongest claim for a completely labile visual framework shifting readily in accordance with the lines of visual organization comes from Wertheimer (39). He had a subject look through a tube into a mirror so placed that the image of the room behind appeared tilted by $45^{\circ}$. At first the room appeared thoroughly disoriented; a man seemed to be walking about on a tilted floor, an object fell in an oblique line, etc But 'after a few minutes' there was a change, he reports, and the room looked upright, with the floor horizontal and the path of falling objects vertical. Wertheimer's interpretation was that the

- The possibility that the simultaneous contrast phenomenon is itself to be regarded as a slight partial shift of all stimulus values on a continuous scale cannot be disregarded. But it seems equally probable that it is an increase of difference between two values on a scale. Cf. Koffka's discussion of color-constancy (24). 
lines of the mirror-image served as 'anchoring forces' determining the subject's vertical and horizontal. His description is unequivocal, although many things one might wish to know about the phenomenon are not stated. A test was made of the subject's ability to set a white line on a circular black ground to the vertical position before and after the main experiment, but these results were not so clear-cut. After the tilted room had righted itself, the line could not be made vertical with the same certainty as before; instead it fluctuated and showed great instability.

Unquestionably, this phenomenon of Wertheimer's supports Koffka's hypothesis that "the main directions of the field constitute the framework" (23, p. 215). The mirrorroom when first seen appears tilted because the subject is still in his normal framework, in which vertical retinal lines produce vertical phenomenal lines. "But this old normal framework has no support in the mirror world and it cannot maintain itself without such support. Instead, the new main lines of organization take over the role of creating the framework: the mirror world right itself" (23, p. 216). In this hypothesis, visual space is labile, relativistic, and completely anchored to, or determined by, processes of visual organization.

One of the writers (Gibson) has repeated Wertheimer's experiment in the effort to verify the phenomenon. A four by six foot mirror at one end of a large room was shifted out of the frontal plane of the subject by rotation around two axes. The top was tilted back and the left end shifted forward. The subject sat looking into this mirror through a cardboard cone fitted to his face which excluded the frame of the mirror but still permitted a $40^{\circ}$ visual field. The mirrorimage of the room was tilted approximately $35^{\circ}$ counterclockwise. Written descriptions were made of several sessions, lasting up to $\mathrm{r} 5$ minutes.

Usually within a minute after the beginning of the observation the tilted room began to look more natural than it had at first. It still looked tilted however, and this character remained unchanged throughout the session. With 
effort, the subject could attempt to project himself into the mirror room and at such times it looked still more naturalless foreign, artificial, or 'wrong.' Such moments could not be maintained for long. The subjective vertical and horizontal were always capable of being called up and used to estimate the inclination of the room. Visually the subject felt himself in or at the end of this room, but the chair on which he sat and the floor which he felt were not a part of it, since they were upright while the room was tilted. The chair and the floor never became congruent with the visual field; the postural 'feel' of the body, no matter how much one might try to neglect it, was an omnipresent factor in the seeing of the room and remained incompatible with it.

At the moments of 'projection' referred to there was a strong involuntary tendency to bend the head in the direction of the room's inclination. If this impulse were yielded to, however, the tilt of the head into alignment with the room seemed to enter into the situation still to prevent the room's looking upright. The tilt of the room persisted, although now changed in character and somewhat decreased in amount. An interpretation of this fact would be that 'perceptual constancy' can preserve the orientation of a tilted environment as well as that of a normal environment. The constancy is incomplete in this situation as it is also in the Aubert Phenomenon.

In order to verify the main results with more subjects and also to try the effect of tilting more lifelike scenes including people and movement, a simple optical system built by the other writer (Mowrer) was employed. It consisted of a pair of totally reflecting right angle prisms mounted in short tubes, one in front of each eye, giving a circular visual field of $28^{\circ}$. The field was reversed from right to left but this fact did not seriously affect the experiment. By rotating both tubes through an appropriate angle, any amount of inclination of the field could be obtained. This device is a simpler version of one used by Brown (5). Three different scenes were observed at degrees of tilt varying from $10^{\circ}$ to $45^{\circ}$. They were (r) a room filled with apparatus seen through a wide doorway, 
(2) a hallway with people entering and leaving doors, and (3) a lawn with trees and part of a building. Five subjects were used although not in all combinations.

The reports completely substantiated the experiment with the mirror. After the first moments when the tilted field appeared unnatural, it began to look real and objects to look familiar. In this state whether the field appeared actually less tilted or only less strikingly tilted was hard to estimate. At any rate the tilt did not disappear; at best it was 'almost not noticed.' Movement of objects in the field seemed to facilitate this condition. No negative after-effect of tilt was reported for the visual field after the conclusion of the experiment, nor any serious instability of the field. ${ }^{4}$

These results are even further confirmed by those of Brown (5), who wore prisms similar to ours for one week, the field being inclined $75^{\circ}$ from the vertical. Although he reports that the tilting of the world was less noticed as time went on, the new situation never became perfectly normal. Spatial behavior became very much easier but the phenomenal inclination persisted.

We are forced to conclude from all this evidence that a tilted visual field does not right itself under the conditions described and that possibly Wertheimer was reporting a relatively more cognitive phenomenon-the decrease in the strangeness and unreality of the mirror-scene. Such an interpretation is compatible not only with the evidence cited but also with the observation of Stratton and Ewert on the complete inversion of the visual field $(35, \mathbf{1 0})$.

In summary, the evidence for the anchorage of the perceived vertical to the $v$-factors consists of these facts: posture is often unstable when vision is eliminated; the perceived vertical, both visual and postural, is often disturbed or even destroyed when the main visual lines move with respect to gravity; and the perceived vertical is partially shifted when

- There is no contradiction involved between these results and Gibson's findings with respect to prolonged inspection of tilted lines. The negative after effects in the latter case seem to be localized shifts relative to the field as a whole, i.e. are confined to the stimulated region of the subjective visual field ( 17 ). 
the main visual lines are tilted by a constant amount (possibly simultaneous contrast). A shift of the perceived vertical into complete congruence with such main lines has not been verified.

\section{Evidence for the Dependance of the Vertical on Gravitational Determinants}

Elimination of visual cues.-Although, as we have seen, there are observations tending to show that the postural and the visual vertical are weakened in the absence of vision, there are on the other hand experiments demonstrating that the postural and the visual vertical are both stable and precise with vision eliminated. The first of these is Garten's investigation (14). Garten built an elaborate tilting-chair on which the subjects sat blindfolded. The chair was first tilted and then set moving slowly toward the normal position; the subject had to stop it when he and the chair felt upright. The accuracy with which this could be done was surprising. After a little practise the average error amounted to less than one degree. The postural vertical, in short, is sensed with great precision. This is the fact in which we are primarily interested. ${ }^{5}$

The visual vertical, too, is sensed with great precision in the absence of any visual cues to anchor the framework. Neal (3I, 29I) found that for subjects in complete darkness, when the framework had been given a presumable opportunity to become unstable, an adjustable luminous line could be set to the vertical with an average variation of only .94 degrees. This figure was based on 720 readings from each of three subjects. She concludes that "localization of the vertical in the dark must depend almost entirely upon the absolute vertical, and the position of the body, and not to any appreci-

sarten went on to isolate the various sensory factors on which this performance depended. He believed that the otolithic function did not play an important part since subjects with defective inner ears were capable of it. And since immersing the subjects' body in water did affect the performance, while anæsthetizing the skin areas in contact with the chair (e.g., cooling the buttocks) did not, he concluded that tactual sensitivity was also eliminated and that the muscle sense is chiefly responsible for this kind of orientation. 
able extent upon points of anchorage in the visual field." The similarity in acuity between Garten's and Neal's results is striking, suggesting once more a common basis for both functions.

Helmholtz (38, p. 256), Jastrow (22), and Gibson and Radner (18), among others, have found that in an illuminated field of view the high acuity for the vertical position of a line (a fraction of one degree) does not depend on the presence of other verticals or horizontals in the field but is a seemingly self-contained function. Furthermore Gibson showed that although the normal retinal-phenomenal correspondence for perception of the vertical may apparently be shifted under the influence of a process similar to sensory adaptation with negative after effect, the maximum change is slight and is confined to the stimulated area (17). The framework as a whole manifests great stability and resistance to such a change in correspondence. ${ }^{6}$

Shift of the direction of gravity so as to conflict with vision.In a series of investigations going back more than a century, a number of observations have been reported concerning the subject's spatial orientation when a horizontal centrifugal force is added to the vertical gravitational force. The first published observations of this kind are apparently those reported by Johann Purkinje (32, p. 88) in 1820 . This writer noted while riding on a carousel, or merry-go-round, that the entire revolving platform seemed somewhat inclined, the point where he was standing appearing lowest and the corresponding point on the opposite side appearing highest. This illusion, says Purkinje, "is so striking and vivid that one involuntarily leans decidedly to one side [toward the center]," in order to bring the long axis of the body into line with the perceived vertical. Purkinje appeared to regard this distortion of the perception of the true vertical as connected in some way with the effect of centrifugal force upon

'It may be noted that, in contrast with adaptation and negative after-effect, the shift involved in maintaining 'constancy' of the framework is instantaneous and completely compensatory. This shift from the normal retinal-phenomenal correspondence which occurs when the retinx are tilted with the head is the more remarkable in view of the resistance to change when normal posture is maintained. 
the blood and the semi-liquid contents of the cranium ( $c f$. Cattell, 8, 1895). More important to us, however, is the observation that the perceived vertical will alter when the direction of gravity is altered. Approximately fifty years after the publication of Purkinje's observations, Ernst Mach (29. pp. 286-287), became much interested in this problem; the circumstances under which his interest was aroused he described in the following words:

I was rounding a sharp railway curve once when I suddenly saw all the trees, houses, and factory chimneys along the track swerve from the vertical and assume a strikingly inclined position. What had hitherto appeared to me perfectly natural, namely, the fact that we distinguish the vertical so perfectly and sharply from every other direction, now struck me as enigmatical. Why is it that the same direction can now appear vertical to me and now cannot? By what is the vertical distinguished for us?

These reflections inspired Mach to construct an apparatus in which he could have himself enclosed in a seated position in a large 'paper box' and uniformly rotated in the horizontal plane several feet removed from the axis of rotation. After the feeling of rotation occasioned by the initial acceleration of the apparatus had had sufficient time to disappear, it seemed to Mach that the box in which he was inclosed was at rest but considerably tilted, the floor of the box sloping downward in the direction away from the axis of rotation, i.e., outward. However, with the stopping of the apparatus, the box seemed promptly to 'straighten up.' The main lines of Mach's visual field-the edges and corners of the box-were apparently ineffective in determining the vertical. On the basis of these observations, Mach concluded (28, p. 27) that whenever a horizontal mass acceleration, due to the centrifugal force, is added to the constant vertical mass acceleration, due to gravity, "one senses the direction of the resultant mass acceleration [which is determined by the law of the parallelogram of forces] and regards this as the vertical."

In his experiment Mach did, however, observe that when 
a pendulum was suspended on the inside of the special 'box' which he employed, the direction of the apparent vertical during uniform rotation usually coincided with the position assumed by the pendulum; but this was not invariably the case. Thus, "it occasionally seemed to me as if the vertical was located between the direction of the pendulum and the axis of my own body" (28, p. 28). Mach apparently attached little significance to this discrepancy, however, and made no attempt to account for it.

There then followed a number of studies, notably those of Delage (9), Kreidl (25), Breuer (3), Breuer and Kreidl (4), Alexander and Barany ( 1 ), and Sachs and Meller (33), in which the problem raised by Purkinje and later by Mach as to which receptors mediate the sensations produced by gravity, or gravity and centrifugal force in combination, was hotly debated. During this period a new factor was introduced into the discussion, namely, the role of the Raddrehung, or counter-rolling, of the eyes which was discovered to be a reflex function of the position of the head with reference to the direction of the pull of gravity. Although this reaction was shown to be primarily dependent upon the vestibular receptors, its discovery introduced a new visual factor into the analysis of the perceived vertical. If, as a result of the existence of a horizontal centrifugal force added to the vertical pull of gravity, the resultant mass acceleration acting on the body occurs in an oblique direction, and if this rotates the eyes reflexly in their sockets so that their vertical meridians tend to come into alignment with the direction of this acceleration, then this distorted position of the eyes might be interpreted as responsible for the tipped appearance of the visual field. This explanation assumes that the perceived vertical should correspond with the vertical meridians of the retinæ-an assumption which was later proved to be false.

In their joint investigation Breuer and Kreidl (4) rotated human subjects in an enclosed compartment at the speed of one revolution in 5.5 seconds and at a distance of 2 meters from the axis of rotation, under which conditions the direction of the resultant mass acceleration acting upon the subjects 
formed an angle of about $15^{\circ}$ with the true vertical. Just prior to the onset of rotation, Breuer and $\mathrm{Kreidl}$ required each subject to fixate a vertical platinum wire, heated to the point of luminosity, for a period sufficiently long to insure a fairly enduring after-image. After the nystagmus which was occasioned by the onset of rotation had disappeared, each subject was required to adjust a pointer on the inside of the compartment in which he was seated so that it coincided with the after-image of the platinum wire. The three subjects used by Breuer and Kreidl showed, on the average, a deviation of the after-images of about $8^{\circ}$. In their experiment the position of the after-images always seemed to coincide perfectly with what appeared to the subjects to be the true vertical. These writers concluded that "it is certain, therefore, that the apparent tilting of the visual field is caused by a real, unconscious counter-rolling (Raddrehung) of the eyes" (4, p. 499). This conclusion is contradicted by subsequent investigations, as we shall see. It is to be noted that the tendency of the perceived vertical to coincide with the changed direction of gravity is only partially effective in Breuer and Kreidl's experiment.

The efforts of the various countries participating in the World War to bring their respective aviation forces to the highest possible point of efficiency resulted in a number of studies of the influence of the different types of aeroplane maneuvers upon the perception of the vertical and of the different sensory mechanisms involved in the efficient piloting of these machines. Observations reported by Head (20), van Wulfften Palthe (37), Leiri (26), Bauer (2), Tschermak and Schubert (36), Gemelli (16), Burtt (6), and others at this time brought out the fact that during properly executed 'turns,' 'spirals,' and even 'loops,' a passenger-if vision of the horizon is excluded-is unable to sense any departure from a normal position with reference to the direction of gravity. When vision is allowed the passenger may either feel that the horizon tilts while the aeroplane retains a constant orientation with respect to the vertical, or that the horizon retains its usual horizontal position, with the aeroplane 
and its cargo undergoing positional changes. Even experienced pilots appear to vary greatly in their reactions in this respect.

Following the World War the problem of the perception of the vertical was again taken back into the laboratory, notably by Garten (I4), Fisher (II, I2, I3), Shoda (34), and Tschermak and Schubert (36). These writers were especially concerned with a re-examination of the theory of Breuer and Kreidl concerning the role of the vestibulo-ocular reflexes in determining the perception of the vertical. In general their conclusions may be represented by the statement of Tschermak and Schubert "that no strict relationship exists between the objective change in eye position and the localization of the apparent vertical" $(36$, p. 240$)$ and by the contention of Garten that the receptors of the non-acustic labyrinth in themselves are probably relatively unimportant for the perception of the vertical.? There is no question, however, of the validity of the original phenomenon discovered by Purkinje and Mach.

Orientation in a 'motordrome.'-A striking opportunity to observe the influence of centrifugal force upon the perception of the vertical (and one which, so far as the writers are aware, has not previously been taken advantage of) is that afforded by the so-called 'motordrome,' where vehicles travel around the inside wall of a large vertical cylinder.

In order to obtain some notion concerning the sensory impressions of persons who perform in these motordromes, one of the writers (Mowrer) has questioned several such

'This is not to say that the non-acustic labyrinth is entirely unrelated to the maintenance of posture and the perception of verticality; it is simply one of the several sensory mechanisms which normally contribute to this complex function. The fact that abnormal stimulation of the labyrinth may rather profoundly disturb equilibration does not, as some writers have supposed, prove that the labyrinth is the sole mediator of the perceptual and postural vertical. It is well known, for example, that if a person is rotated for a time with the head, let us say, tilted sharply forward and is then told upon stopping to raise the head, a violent 'falling reaction' to one side and accompanying disturbances of the perceived vertical will ensue. But it is quite as fallacious to conclude on the basis of this or similar facts that the labyrinthine receptors are the exclusive mediators of posture-determining stimuli as it would be to conclude from sorne of the other observations cited above that the eyes are alone important in this connection. 
persons and on two occasions has accompanied one of them. In a 'drome' with a diameter of 40 feet, a motorcycle traveling around the vertical sidewall at a speed of $35 \mathrm{~m}$.p.h. is acted upon by a horizontal centrifugal force equal to approximately 3.9 times gravity. Naturally one's 'weight' under such conditions is relatively tremendous; the task of lifting a hand (which feels as if it might be made of lead) involves exorbitant effort. At still greater speeds the blood is centrifuged from the head, with ensuing cerebral enemia and loss of consciousness, the occurrence of which is presaged by what the riders refer to as 'going blind.' Following a ride in a motordrome there is also an interesting form of postrotational nystagmus which, however, cannot be pertinently discussed here.

Without exception performers in a motordrome report the feeling that they are riding in a virtually upright position, with the motordrome lying on its side and rolling along barrel-fashion. The actually vertical wall of the motordrome 'beneath' them is perceived as perfectly horizontal. Therefore, since the vector resultant formed by centrifugal force and gravity is not quite perpendicular to the wall of the motordrome (deviating about $14^{\circ}$ at a speed of $35 \mathrm{~m} . \mathrm{p} . \mathrm{h}$. in a 'drome' of the size indicated), a motorcycle rider will feel that he is tilted slightly away from his perceived vertical, which is perpendicular to the wall. Experienced riders learn to accept this unnatural position, but for the neophyte it is extremely disconcerting, causing him continually to be trying to 'straighten up' (i.e., sit in an actually horizontal position) which of course interferes with the balancing of the motorcycle.

On the first occasion when the writer accompanied a professional rider in a motordrome, the only describable experience was one of complete spatial disorientation. Movement was clearly perceptible but it was impossible to give it any definite directional reference. It was as if a complexly integrated perceptual mechanism had completely broken down; and this, we conjecture, is precisely what had happened. Visual and postural factors were thrown into such violent conflict that no stable perceptual organization could emerge. 
However, on the occasion of the second ride (which occurred only a few minutes after the first), this confusion disappeared, and the spatial illusion reported by veteran riders was vividly experienced.

Here it is significant to note that although this illusion, that the motordrome is lying on its side and revolving in this position, clearly reveals the predominance of what we have called gravitational factors over visual factors, nevertheless visual factors exert some influence on the perceived vertical. If the g-factors were the sole determinants in this situation the rider's apparent vertical would deviate just $76^{\circ}$ from the real vertical, coinciding with the vector resultant. As it is, the rider's vertical deviates $90^{\circ}$ from the real vertical, presumably under the influence of a visual tendency for the sidewall to determine the rider's horizontal. In other words we are accustomed to ride in a visually upright position on a horizontal surface, and within limits this visual factor exerts its own influence. A new perceptual integration is achieved, in which gravitational factors are the primary determinants but which is also influenced to some degree by visual cues. ${ }^{8}$

\section{Discussion}

The evidence which has been summarized would seem to support the hypothesis that both the framework of visual space and postural orientation are jointly determined by both visual and gravitational factors, but that the latter are more decisive. Not only do the facts point to this conclusion but also there is an argument for it which we have not yet brought to bear. If one goes back and examines the two lists of stimuli outlined at the beginning of the paper, the g-factors and the $v$-factors, it becomes evident that the visual stimuli can have specificity for either postural or phenomenal response-processes only if a fixed posture of the eyes is established. Visual lines are not in their own right stimuli for orientation.

B It must be kept in mind that the intensity of the total mass acceleration acting upon the body in a motordrome is several times greater than normal gravity. Because of this augmentation of intensity, it is possible that spatial perception is here influenced somewhat more by what we have called gravitational factors than would be the case if the direction of gravity could be changed without also being intensified. 
If the eyes rolled at random within the head, if the organism could not be oriented to gravity, a vertical line of stimulation on the retina would be neurologically meaningless. Logically, therefore, visual orientation presupposes postural orientation. Once orientation to gravity is granted, visual cues can make their contribution both to posture and to the uprightness of seen space; they become specific. Only at this stage of reasoning does it become possible to postulate a mechanism for the automatic correction of visual cues in conditions of sensed abnormal posture. The visual framework can now be subject to 'constancy,' or in other words, tilted retinal lines can yield upright phenomenal lines when the head is correspondingly tilted. Once there is specificity of stimuli there can be orderly variations in this specificity, but not before. The priority of the g-factors to the $\mathrm{v}$-factors is thus a logical matter. That they are also genetically prior is indicated by the fact that geotropisms precede pattern-vision in the evolution of sensory functions.

Vision 'fills in' the framework, as it were, but its skeleton tends to be fixed by gravity. Undoubtedly visual patternstimulation has some secondary part in helping to 'anchor' visual space but its basic anchorage is to the postural vertical. Spatial perception, in other words, is mediated by postural mechanisms. And this is necessarily so, since essentially what we are saying is that there can be no phenomenal space without posture.

A fundamental difficulty with the older theories of spaceperception was that they strove first to explain how space could be sensed, experienced, or seen-what the 'cues' were for perception-and then on the basis of these experiences the appropriate spatial behavior was supposed to be explicable. But if perceptual space is a motor phenomenon through and through, if the spatial experiences and sensed relations are reflections of discriminative response just as truly as the other way around, then this line of attack was a mistaken one. It may be argued that sensed cues, judgments, or experiences should not be made the basis for spatial behavior for the reason that they are themselves already spatial behavior. 
A man who crosses a stream by jumping from rock to rock without wetting his feet illustrates a psychological performance whose perceptual and behavioral elements are inseparable. It should not be accounted for in terms merely of his correct sense of the vertical or of his excellent equilibritory reactions for, properly considered, these are both abstract features of a unitary function in which the behavior is not merely spatial but the space is behavioral.

\section{Conclusions}

I. The vertical and horizontal axes of perceived space have no empirical meaning except with reference ultimately to the direction of terrestrial gravity.

II. Certain visual lines (the main lines of the visual field) normally bear a fixed relation to the direction of gravity.

III. Phenomenal uprightness is a complex feature of perception, dependent upon the integration of variable visual and postural (gravitational) determinants.

IV. When the head is upright with respect to gravity (or tilted so little that the reflex counter-rolling of the eyes adequately compensates for the tilt) the vertical and horizontal lines of stimulus-objects fall upon the vertical and horizontal meridians of the retinæ; when the head is tilted markedly this congruence is destroyed, yet there is no distortion of the perceived vertical (the constancy phenomenon). Presumably this results from the concomitant alteration of postural stimuli.

$V$. When the main lines of the visual field are eliminated, the postural vertical may sometimes be disturbed; nevertheless under experimental conditions both the postural and the visual vertical are sensed with a high degree of acuity in the absence of vision.

VI. When the main lines of the visual field are artificially shifted with respect to gravity by a fixed amount, a corresponding shift of the perceived vertical may occur; but the shift is relatively slight and the visual field does not, as has been claimed, 'right itself' under these conditions.

VII. When the main lines of the visual field are shifted 
with respect to gravity more or less continuously (remain in motion), instability of the perceived vertical is likely to occur and to be conspicuous.

VIII. When the direction of the pull of gravity is considerably altered by the addition of a centrifugal force and the main visual lines remain unaltered, a deviation of the perceived vertical always occurs by an amount which sometimes approaches the degree of alteration.

IX. When the direction of gravity and the main visual lines are both concomitantly altered, in the same direction and by the same amount (as in a 'banking' train or cabin airplane), the perceived vertical deviates correspondingly by the same amount, as would be expected.

X. Gravitational (postural) factors are both logically and psychologically prior to visual factors in the determination of the perceived vertical. The framework of visual space is derived from and dependent upon the necessity of maintaining postural equilibrium against the pull of gravity.

\section{REFERENCES}

I. Alexander, G. und Bárány, R., Psychophysiologische Untersuchungen uber die Bedeutung des Statolithenapparates für die Orientierung im Raum an Normalen und Taubstummen, nebst Beiträgen zur Orientierung mittels taktiler und optischer Empfindungen, Zsch. f. Psychol. u. Physzol. d. Sinnes., 1904, 37, 321-362 and $414-457$.

2. Bauer, L. H., Dangers of high speed and acrobatic flying, Aoiation, 1924, 16, $174-176$.

3. Breuer, J., Ueber die Function der Otolithen-Apparate, Pfüg. Arch. f. d. ges. Physiol., 1891, 48, 195-306.

4. - UND KREIDL, A., Ueber die scheinbare Drehung des Gesichtsfeldes wahrend der Einwirkung einer Centrifugalkraft, Pfiug. Arch. f. d. ges. Physiol., 18g8, 70, 494-507.

5. Brown, G. G., Perception of depth with disoriented vision, Brit. J. Psychol., 1928, $29,117-146$.

6. Burt, H. E., The perception of slight changes of equilibrium, with especial reference to problems of aviation, J. Appl. Psychol., 1918, 2, 101-115.

7. CARR, H. A., An introduction to space perception, New York: Longmans, Green, 1935, Chap. 9.

8. Cattell, J. McK., The sense of equilibrium, Science, 1895, 2 (new series), 99-100.

9. Delage, Y., Etudes expérimentales sur les illusions statiques et dynamiques de direction pour servir a déterminer les fonctions des canaux semicirculaires de l'oreille interne, Arch. d. zool. expér. et gén., I 886, 4, 535-624.

10. EwERT, P. H., A study of the effect of inverted retinal stimulation upon spatially coordinated behavior, Genet. Psychol. Monog., 1930, 7, 177-363. 
II. Fischer, M. H., Messende Untersuchungen über die Gegenrollung der Augen und die Lokalisation der scheinbaren Vertikalen bei seitlicher Neigung (des Kopfes, des Stammes and des Gesamtkörpers), von Graefes Arch. f. Ophthalmol., $1927,118,633-680$.

12. - Die Regulationsfunktionen des menschlichen Labyrinthes und Zusammenhange mit verwandten Funktionen, Ergeb. der Physzol, 1928, 27, 209-379.

13. - Messende Untersuchungen über die Gegenrollung der Augen und die Lokalisation der scheinbaren Vertikalen bei seitlicher Neigung des Gesamtkorpers bis zu $360^{\circ}$. II Undersuchungen an Normalen, von Graefes Arch. f. Ophthalmol., $1930,123,476-508$.

14. Garten, S., Über die Grundlagen unserer Orientierung im Raume, Abhandl. d. Math.-Phys. Klasse de Sächs. Akad., 1920, 36, 431-510.

15. GelB, A., Die psychologische Bedeutung pathologischer Storungen der Raumwahrnchmungen, Jena: Ber. ub. d. IX Kongr. f. Exp. Psychol., 1926, 28-32.

16. Gemelli, A., L'orientazione lontana vel volo in aroplano, Rio. di psicol., I933, 29, 297-325.

17. Gibson, J. J., Adaptation, after-effect, and contrast in the perception of tilted lines. II. Simultaneous contrast and the areal restriction of the after-effect, J. Exper. Psychol., 1937, 20, 553-569.

I8. - ANd Radner, M., Adaptation, after-effect, and contrast in the perception of tilted lines. I. Quantitative studies, J. Exper. Psychol., 1937, 20, 453-467.

19. - and Robinson, D., Orientation in visual perception; the recognition of familiar plane forms in differing orientations, Psychol. Monog., 1935, No. 210, $39-47$.

20. Head, H., The sense of stability and balance in the air, Reports of the Medical Research Committee of Great Britain, I918.

21. JAckson, E., Vision for equilibrium and orientation, Amer. J. Ophthal., 1933, 16, $415 \mathrm{ff}$.

22. Jastrow, J., On the judgment of angles and positions of lines, Amer. J. Psychol., $1893,5,220 \mathrm{ff}$.

23. KoffKA, K., Gestalt psychology, New York: Harcourt Brace, 1935.

24. - Some remarks on the theory of color constancy, Psychol. Forsch., 1932, 16, 329-353.

25. KReIDL, A., Beitràge zur Physiologie des Ohrlabyrinths auf Grund von Versuchen an Taubstummen, Pflug. Arch. f.d. ges. Physiol., 1892, 51, I17-150.

26. LeIRI, F., Uber die Bedeutung des Vestibularapparats bei der aviation, Zsch. $f$. Hals-, Nasen-, und Ohrenheilkunde, 1927, 17, 381-391.

27. MACH, E., Physikalische Versuche über den Gleichgewichtssinn des Menschen, Sitzungsberichte der kaiserl. Akad. der Wissens., 1873, 68, 124-140.

28. - Grundlinien der Lehre von den Bewegungsempfindungen, Leipzig: Wilhelm Engelmann, 1875, pp. 128.

29. - Popular Scientific Lectures (Trans. by T. J. McCormack), Chicago: Open Court, 1898, 282-308.

30. Muller, G. E., Über die Aubertsche Phänomen, Zsch. f. Sinnesphysiol., 1916, 49, $109-244$.

31. Neal, E., Visual localization of the vertical, Amer. J. Psychol., 1926, 37, 287-291.

32. Purkinje, J., Beiträge zur nảheren Kenntniss des Schwindels aus herautognostischen Daten, Wien: Medicinische Jahrbucher des katserl. königl. österreichischen Staates, 1820, 4 (ii), 79-125. 
33. SAchs, M. UNd Meller, J., Untersuchen über die optische und haptische Lokalisation bei Neigungen um eine sagittale Achse, Zsch. f. Prychol. und Physiol. der Sinnes., 1903, 31, 89-109.

34. Shoda, M., Uber die scheinbare Vertikale bei Tertiarstellung des Auges, Pfĭg. Arch. f. d. ges. Physiol., 1926, 215, 588-607.

35. Stratton, G. M., Vision without inversion of the retinal image, Psycnor. Rev., $1896,3,611-617 ; 1897,4,341-360,463-481$.

36. Tschermax, A. und Schubert, G., Uber Vertikalorientierung im Rotatorium und im Flugzeuge, Pflug. Arch.f.d. ges. Physiol., 1931, 228, 234-257.

37. vas Wulfrten Paltee, P M., Function of the deeper sensibility and of the vestibular organs in flying, Acta Otolaryngologica, 1922, 4, 415-449.

38. Von Helmholtz, H., Physiological Optics (Trans. by J. P. C. Southall), vol. 3. 39. Wertheimer, M., Experimentelle Studien über das Sehen von Bewegung, Zsch. f. Psychol., 1912, 6r, $257 \mathrm{ff}$.

40. WoOd, H. C., The 'haunted swing' illusion, Psychol. Rev., 1895, 2, 277-278.

[MS. received November 15, 1937] 